\title{
Massive gravitons dark matter scenario revisited
}

\author{
Hyung Won Lee $*$ Kyoung Yee Kim $t+$ and Yun Soo Myung $\sharp$ \\ Institute of Basic Science and School of Computer Aided Science, \\ Inje University, Gimhae 621-749, Korea
}

\begin{abstract}
We reexamine the massive graviton dark matter scenario (MGCDM) which was recently considered as an alternative to dark energy models. When introducing the native and effective equations of state (EoS), it is shown that there is no phantom phase in the evolution toward the far past. Also we show that the past accelerating phase arises from the interaction between massive graviton and cold dark matter.

PACS numbers: 98.80.-k, 95.35.+d, 95.36.+x
\end{abstract}

*Electronic address: hwlee@inje.ac.kr

${ }^{\dagger}$ Electronic address: kimky@inje.ac.kr

${ }^{\ddagger}$ Electronic address: ysmyung@inje.ac.kr 


\section{INTRODUCTION}

Recently the massive graviton dark matter scenario (MGCDM) which has been originally proposed by Visser [1] was considered as an alternative dark energy model to explaining the present accelerating universe [2, 3]. In order to explain the evolution of the universe, they used the "geometric" dark energy EoS mainly as [3]

$$
w_{\mathrm{DE}}(a)=-1-\frac{1}{3} \frac{d \ln \delta H^{2}}{d \ln a}=-1-\frac{2}{3}\left(\frac{7-10 a^{2}}{7-5 a^{2}}\right),
$$

which shows that the massive graviton theory can be treated as an additional effective fluid with EoS (11) cosmologically. Also they have emphasized that the current Hubble function has only two free parameters of $H_{0}$ and $\Omega_{m}$ which are the same number of parameters as the $\Lambda$ CDM model. However, this MGCDM showed a different phantom phase of $\lim _{a \rightarrow 0} w_{\mathrm{DE}}(a)=-5 / 2$ and a current decelerating phase [3], in compared to the $\Lambda \mathrm{CDM}$ model with $w_{\Lambda}=-1$. This is mainly because they used the geometric dark energy EoS $w_{\mathrm{DE}}(a)$.

On the other hand, it is well known that if the conservation law is modified due to the

presence of other matter as ([6) $)$, one has to introduce the effective EoS parameter $w_{a}^{\text {eff }}(a)$ as well as the native $\operatorname{EoS} w_{g}^{\text {nat }}(a)$ to describe the evolution of the universe correctly [4]. For the holographic dark energy model, two of authors have clarified that although there is a phantom phase when using the native EoS [5], there is no phantom phase when using the effective EoS [6, 7].

In this work, we wish to reexamine the evolution of the universe based on the MGCDM by introducing the native and effective EoS. It is hard to derive any phantom phase when using the effective EoS $w_{g}^{\text {eff }}$ instead of the geometric EoS $w_{\mathrm{DE}}$. Finally, we show that the past accelerating phase arises from the interaction between massive graviton and CDM.

\section{MASSIVE GRAVITON}

We briefly review the Visser's massive gravity approach [1]. The action is given by

$$
S=\int d^{4} x\left[\sqrt{-g} \frac{c^{4} R(g)}{16 \pi G}+\mathcal{L}_{\text {mass }}\left(g, g_{0}\right)+\mathcal{L}_{\text {matter }}(g)\right]
$$

where the first term is the Einstein-Hilbert Lagrangian and the last is the Lagrangian of matter. The second term is designed for the massive graviton expressed in terms of the 
bi-metric $\left(g_{0}, g\right)$ as

$$
\begin{aligned}
\mathcal{L}_{\text {mass }}\left(g, g_{0}\right)= & \frac{1}{2} \frac{m_{g}{ }^{2} c^{2}}{\hbar^{2}} \sqrt{-g_{0}}\left\{\left(g_{0}^{-1}\right)^{\mu \nu}\left(g-g_{0}\right)_{\mu \sigma}\left(g_{0}^{-1}\right)^{\sigma \rho}\right. \\
& \left.\times\left(g-g_{0}\right)_{\rho \nu}-\frac{1}{2}\left[\left(g_{0}^{-1}\right)^{\mu \nu}\left(g-g_{0}\right)_{\mu \nu}\right]^{2}\right\},
\end{aligned}
$$

where $m_{g}$ is the graviton mass and $\left(g_{0}\right)_{\mu \nu}$ is a general flat metric.

The Einstein equations takes the form

$$
G^{\mu \nu}-\frac{1}{2} \frac{m_{g}{ }^{2} c^{2}}{\hbar^{2}} M^{\mu \nu}=-\frac{8 \pi G}{c^{4}} T^{\mu \nu}
$$

where $G^{\mu \nu}$ is the Einstein tensor, $T^{\mu \nu}$ is the energy-momentum tensor for a perfect fluid, and

$$
M^{\mu \nu}=\left(g_{0}^{-1}\right)^{\mu \sigma}\left[\left(g-g_{0}\right)_{\sigma \rho}-\frac{1}{2}\left(g_{0}\right)_{\sigma \rho}\left(g_{0}^{-1}\right)^{\alpha \beta} \times\left(g-g_{0}\right)_{\alpha \beta}\right]\left(g_{0}^{-1}\right)^{\rho \nu} .
$$

In the limit $m_{g} \rightarrow 0$, we recover the Einstein equation is recovered. Importantly, since the Einstein tensor should satisfy the Bianchi identity $\nabla_{\nu} G^{\mu \nu}=0$, the non-conservation law of the energy-momentum tensor is obtained as

$$
\nabla_{\nu} T^{\mu \nu}=\frac{m_{g}^{2} c^{6}}{16 \pi G \hbar^{2}} \nabla_{\nu} M^{\mu \nu}
$$

which will play the crucial role in the cosmological evolution.

\section{COSMOLOGICAL EVOLUTION}

In order to apply the action (2) to cosmology, we need to introduce the bi-metric explicitly. First we use the Friedmann-Roberston-Walker metric as the dynamical one

$$
d s_{\mathrm{FRW}}^{2}=c^{2} d t^{2}-a^{2}(t)\left[d r^{2}+r^{2} d \Omega_{2}^{2}\right]
$$

with $a(t)$ the scale factor. The flat metric expressed in terms of spherical coordinates is proposed to be the static one:

$$
d s_{0}^{2}=c^{2} d t^{2}-\left[d r^{2}+r^{2} d \Omega_{2}^{2}\right]
$$

The evolution of a MGCDM cosmology is governed by two Friedmann equations:

$$
\left(\frac{\dot{a}}{a}\right)^{2}=\frac{8 \pi G}{3 c^{2}} \rho+\frac{m_{g}{ }^{2} c^{4}}{4 \hbar^{2}}\left(1-a^{2}\right)
$$




$$
\frac{\ddot{a}}{a}+\frac{1}{2}\left(\frac{\dot{a}}{a}\right)^{2}=-\frac{4 \pi G}{c^{2}} p-\frac{m_{g}{ }^{2} c^{4}}{8 \hbar^{2}} a^{2}\left(a^{2}-1\right),
$$

where $\rho$ is the energy density and $p$ is the pressure. From the observation of Eqs. (9) and (10), one can read off the energy density and pressure for massive graviton as

$$
\begin{gathered}
\rho_{g}=\frac{3 m_{g}^{2} c^{6}}{32 \pi G \hbar^{2}}\left(1-a^{2}\right), \\
p_{g}=\frac{m_{g}^{2} c^{6}}{32 \pi G \hbar^{2}} a^{2}\left(a^{2}-1\right) .
\end{gathered}
$$

We note that the positive energy density and negative pressure is allowed to explain the accelerating universe. In this case, it requires

$$
a^{2}<1
$$

Hence the native equation of state is simply given by

$$
w_{g}^{\mathrm{nat}}(a) \equiv \frac{p_{g}}{\rho_{g}}=-\frac{1}{3} a^{2}
$$

whose limit of $a \rightarrow 0$ is zero. From (6) , we find the the non-conservation law for the matter including cold dark matter (CDM)

$$
\dot{\rho}+3 H(\rho+p)=-3 H \frac{m_{g}{ }^{2} c^{6}}{32 \pi G \hbar^{2}}\left(a^{4}-6 a^{2}+3\right) \equiv-3 H Q
$$

with $H=\dot{a} / a$ the Hubble parameter and

$$
Q=\frac{m_{g}^{2} c^{6}}{32 \pi G \hbar^{2}}\left(a^{4}-6 a^{2}+3\right)
$$

On the other hand, one can obtain the non-conservation law for massive graviton by computing (11) and (12) directly

$$
\dot{\rho_{g}}+3 H\left(\rho_{g}+p_{g}\right)=3 H Q
$$

Rewriting Eq. (17) as

$$
\dot{\rho}_{g}+3 H\left(1+w_{g}^{\mathrm{eff}}\right) \rho_{g}=0
$$

we can define the effective equation of state for massive graviton [4]

$$
w_{g}^{\mathrm{eff}}(a)=-\frac{1-\frac{5}{3} a^{2}}{1-a^{2}}
$$


whose limit of $a \rightarrow 0$ is -1 . When introducing the massive graviton $\mathcal{L}_{\text {mass }}$, both matter and massive graviton do not satisfy their own conservation law as is shown by (15) and (17). Instead, the total mixture of matter and massive graviton fluid satisfies the conservation law as

$$
\dot{\rho}_{t}+3 H\left(\rho_{t}+p_{t}\right)=0
$$

with $\rho_{t}=\rho+\rho_{g}$ and $p_{t}=p+p_{g}$. This shows clearly that our picture is quite different from (1) imposed by Ref.[2, 3]. In other words, in order to express the evolution of universe due to the massive graviton properly, we will use its own native $\operatorname{EoS} w_{g}^{\text {nat }}$ and effective $\operatorname{EoS} w_{g}^{\text {eff }}$ instead of $w_{\mathrm{DE}}$. This is a well-accepted approach to cosmology when two different matters coexist in the universe, showing that either decaying of massive graviton to CDM for $Q<0$ or decaying of CDM to massive graviton for $Q>0$ as in quintessence [4], $\Lambda(t) \mathrm{CDM}$ model [8], and the Brans-Dicke cosmology [9].

Now we are in a position to solve Eq. (9) for $a(t)$. For this purpose, Eq. (9) can be written as

$$
H^{2}=H_{0}^{2}\left[\chi \Omega_{m}^{0}\left(\frac{a_{0}}{a}\right)^{4}+\Omega_{m}^{0}\left(\frac{a_{0}}{a}\right)^{3}+\frac{\alpha_{g}^{2}}{4}\left(1-a^{2}\right)\right],
$$

where $H_{0}$ is the Hubble constant at current, $\chi$ is the current ratio of radiation density to dark matter density, and $\Omega_{m}^{0}$ is the current density parameter of CDM. $\alpha_{g}$ is the ratio of graviton mass and Hubble mass

$$
\alpha_{g}=\frac{m_{g}}{m_{H}}, \quad m_{H}=\frac{\hbar H_{0}}{c^{2}}=3.8026 \times 10^{-69} h_{0} \mathrm{~kg},
$$

where $h_{0}$ is defined as $H_{0}=100 h_{0} \mathrm{~km} / \mathrm{s} / \mathrm{Mpc}$. Evaluating Eq. (21) at the present time, the current scale factor is determined to be

$$
a_{0}=\sqrt{1-\frac{4}{\alpha_{g}^{2}}\left(1-\Omega_{m}^{0}-\chi \Omega_{m}^{0}\right)} .
$$

In order to have real value for $a_{0}$, one requires

$$
\alpha_{g}>2 \sqrt{1-\Omega_{m}^{0}-\chi \Omega_{m}^{0}}
$$

which corresponds to the inequality on the mass of massive graviton as

$$
m_{g}>2 \sqrt{1-\Omega_{m}^{0}-\chi \Omega_{m}^{0}} m_{H}
$$

Taking $\Omega_{m}^{0}=0.27$ and $\chi \simeq 3.1 \times 10^{-4}$, we have two bounds on $\alpha_{g}$ and $m_{g}$

$$
\alpha_{g}>1.71, m_{g}>1.71 m_{H} \simeq 6.50 \times 10^{-69} h_{0} \mathrm{~kg}
$$




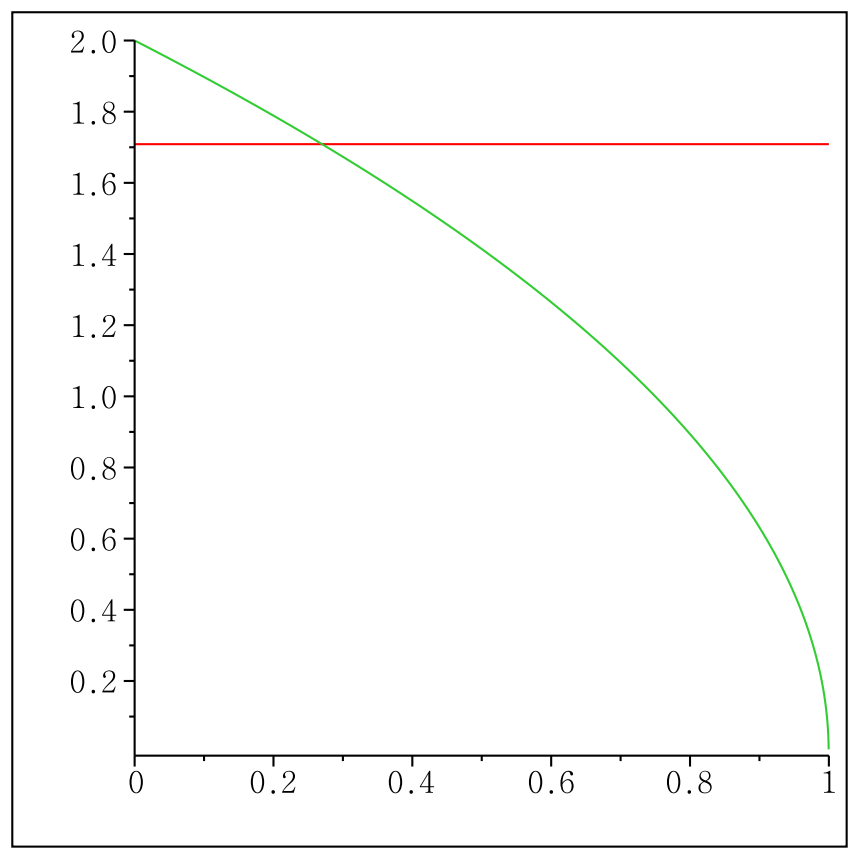

FIG. 1: $\alpha_{g}$ as a function of current matter density parameter, $\Omega_{m}^{0}$. Horizontal line represents its current value $\alpha_{g}\left(\Omega_{m}^{0}\right)=1.71$ at $\Omega_{m}^{0}=0.27$.

$\alpha_{g}$ is depicted as a function of $\Omega_{m}^{0}$ in Fig. 1 and its current value is $\alpha_{g}\left(\Omega_{m}^{0}\right)=1.71$.

At this stage, we introduce density parameters

$$
\Omega_{m}=\frac{8 \pi G}{3 H^{2} c^{2}} \rho_{m}, \quad \Omega_{r}=\frac{8 \pi G}{3 H^{2} c^{2}} \rho_{r}, \quad \Omega_{g}=\frac{8 \pi G}{3 H^{2} c^{2}} \rho_{g} .
$$

Solving the Friedmann equations (21) together with the conservation laws (15) and (17), the relevant cosmological parameters are determined to be

$$
\begin{gathered}
\Omega_{r}=\frac{\chi \Omega_{m}^{0} e^{-4 x}}{\chi \Omega_{m}^{0} e^{-4 x}+\Omega_{m}^{0} e^{-3 x}+\frac{\alpha_{g}^{2}}{4}\left(1-a_{0}^{2} e^{2 x}\right)}, \\
\Omega_{m}=\frac{\Omega_{m}^{0} e^{-3 x}}{\chi \Omega_{m}^{0} e^{-4 x}+\Omega_{m}^{0} e^{-3 x}+\frac{\alpha_{g}^{2}}{4}\left(1-a_{0}^{2} e^{2 x}\right)}, \\
\Omega_{g}=\frac{\alpha_{g}^{2}}{4} \frac{1-a_{0}^{2} e^{2 x}}{\chi \Omega_{m}^{0} e^{-4 x}+\Omega_{m}^{0} e^{-3 x}+\frac{\alpha_{g}^{2}}{4}\left(1-a_{0}^{2} e^{2 x}\right)}, \\
H^{2}=H_{0}^{2}\left[\chi \Omega_{m}^{0} e^{-4 x}+\Omega_{m}^{0} e^{-3 x}+\frac{\alpha_{g}^{2}}{4}\left(1-a_{0}^{2} e^{2 x}\right)\right] \\
w_{g}^{\text {nat }}=-\frac{a_{0}^{2} e^{2 x}}{3} \\
w_{g}^{\mathrm{eff}}=-\frac{1-\frac{5}{3} a_{0}^{2} e^{2 x}}{1-a_{0}^{2} e^{2 x}}
\end{gathered}
$$


with a new variable $x=\ln \left(a / a_{0}\right) \in[-\infty, \infty]$ including $x=0$ at $a=a_{0}$, instead of scale factor $a$. The time evolution of all parameters including density parameters, EoS, and Hubble parameter is shown as a function of $x$ in Fig. 2. This graphs indicates that there is no phantom phase when using the native $\operatorname{EoS} w_{g}^{\text {nat }}$ and the effective EoS $w_{g}^{\text {eff }}$ but there is a phantom phase when using the geometric EoS $w_{\mathrm{DE}}$. This shows clearly that the description with $w_{\mathrm{DE}}$ is not appropriate for interpreting the modified evolution due to the massive graviton.

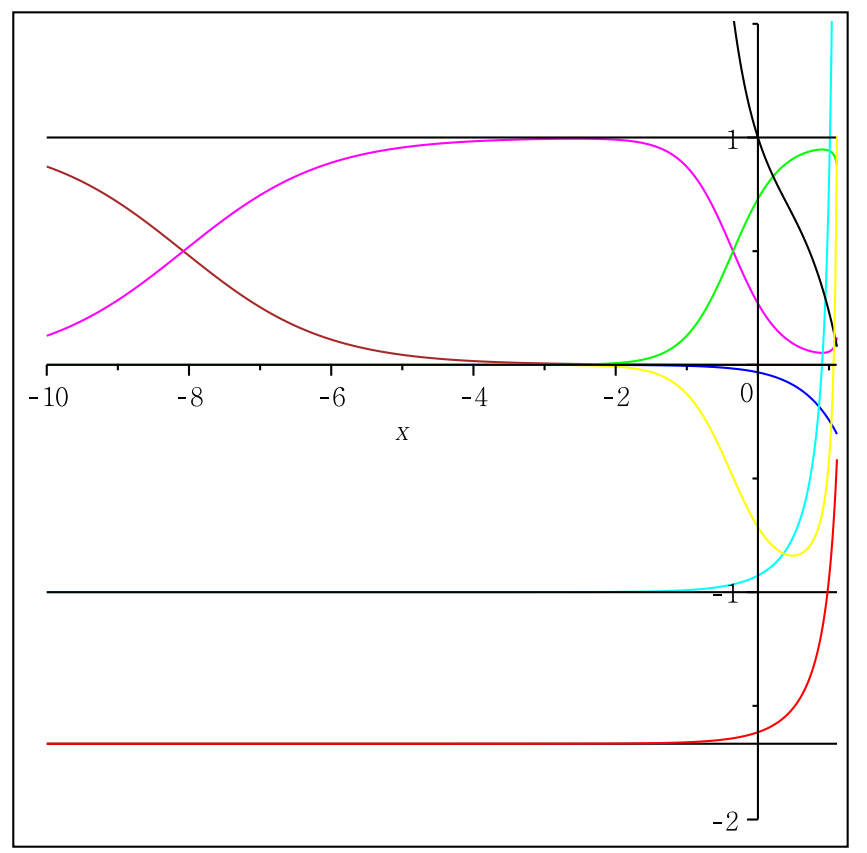

FIG. 2: Cosmological evolution for there density parameters $\left[\Omega_{g}\right.$ (green),$\Omega_{m}$ (magenta), $\Omega_{r}($ brown$\left.)\right]$, four equations of states $\left[w_{g}^{\text {nat }}(\right.$ blue $), w_{g}^{\text {eff }}(\operatorname{cyan}), w_{\mathrm{DE}}($ red $), w^{\text {eff }}($ yellow $\left.[2])\right]$, and Hubble parameter (black) as function of $x$, for $\Omega_{m}^{0}=0.27, \chi=3.1 \times 10^{-4}$, and $\alpha_{g}=1.8$. The bottom line corresponds to asymptote of $-5 / 3$ to $w_{\mathrm{DE}}$ shown in Ref.[3], while the second lowest line represents asymptote of -1 to $w_{g}^{\text {eff }}$.

In order to compute the age of the universe, we simply evaluate the integration

$$
t_{U}=\int_{0}^{a_{0}} \frac{d a}{a H(a)}
$$

Substituting (21) in (34) leads to the age of the universe as shown Fig. 3 for chosen values of $\Omega_{m}^{0}$, showing that the age of the universe is closely related to the mass of massive graviton. We note that $H_{0}^{-1}=10.0 h_{0}^{-1} \mathrm{~Gy}$. 


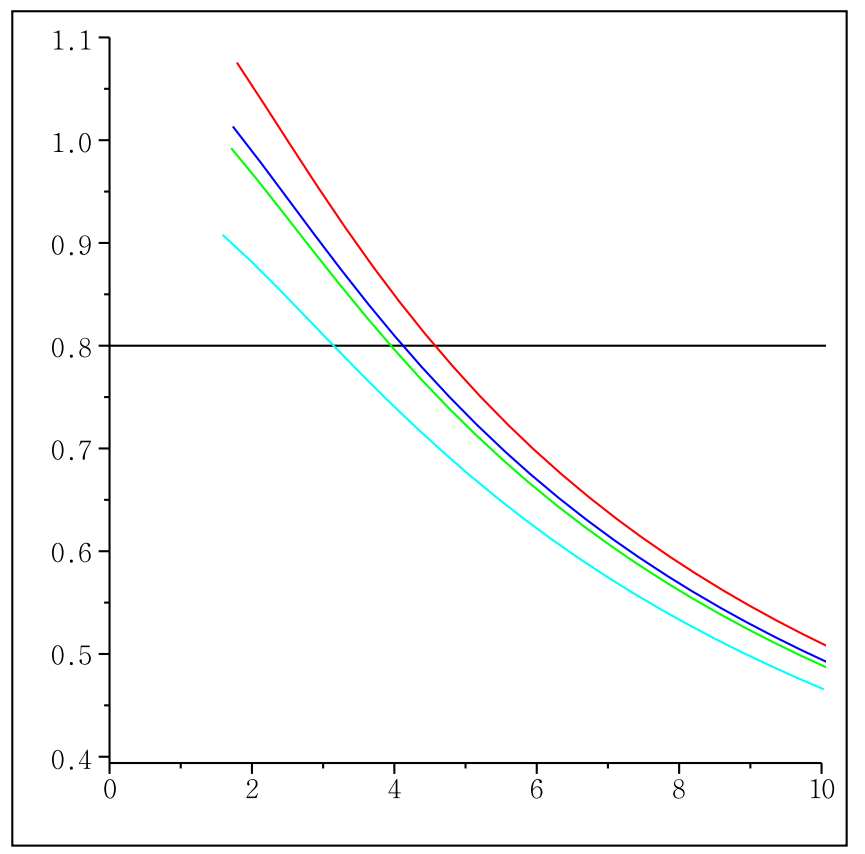

FIG. 3: Relation between the age $t_{U}$ of the universe and the mass ratio $\alpha_{g}$ for different values of $\Omega_{m}^{0}$, 0.20(red), 0.25(blue), 0.27(green), 0.37(cyan), and horizontal line denotes 0.8, which corresponds to $8 h_{0}^{-1}$ Gy.

At this stage, we would like to mention that as is shown in Eq. (21), the Hubble parameter becomes zero at a certain value of $a_{c}$ satisfying the condition of

$$
\chi \Omega_{m}^{0}\left(\frac{a_{0}}{a_{c}}\right)^{4}+\Omega_{m}^{0}\left(\frac{a_{0}}{a_{c}}\right)^{3}+\frac{\alpha_{g}^{2}}{4}\left(1-a_{c}^{2}\right)=0 .
$$

If one chooses $\alpha_{g}$ as the saturating bound in Eq. (24), $a_{0}$ becomes zero from Eq. (23) and consequently, (35) implies that $a_{c}$ becomes 1 . However, this case is meaningless because the current scale factor is chosen to be zero. Hence $\alpha_{g}$ should satisfy the bound of Eq. (24). This implies that the mass of massive graviton should be greater than a certain value to have a non-zero scale factor $\left(m_{g}>1.71 m_{H}\right)$ at current time. In this case, we have the condition of $a_{c}>1$ which means that the energy density of massive graviton is negative as is shown in (11). In connection to this point, we may solve Eq. (21) for $a$ as a function of cosmological time. The result is depicted in Fig. 4 for $\alpha_{g}=1.8, \Omega_{m}^{0}=0.27$, and $\chi=3.1 \times 10^{-4}$, indicating that there is no sizable difference when comparing to other cases.

Finally, we derive the geometric equation of state $w_{\mathrm{DE}}$ in (1) from our approach. We 


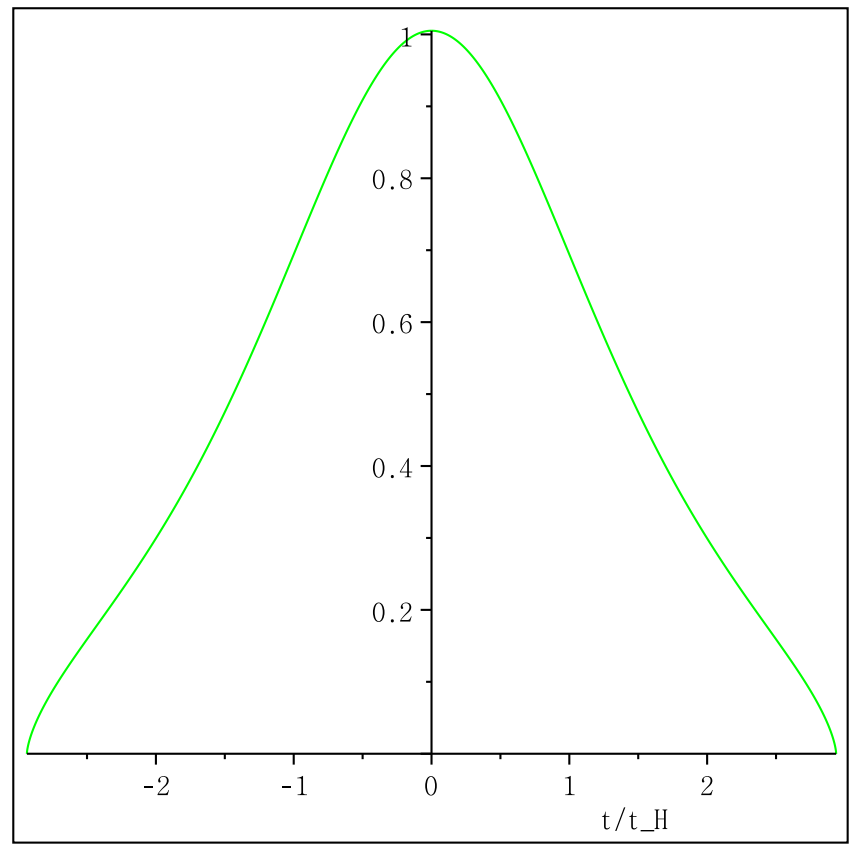

FIG. 4: Time evolution for scale factor $a$ for $\alpha_{g}=1.8, \Omega_{m}^{0}=0.27$, and $\chi=3.1 \times 10^{-4}$.

start with the modified Hubble equation

$$
H^{2}=H_{0}^{2}\left[\Omega_{m}^{0}\left(\frac{a_{0}}{a}\right)^{3}+\delta H^{2}\right]=H_{m}^{2}+H_{\delta}^{2},
$$

where $H_{\delta}^{2}$ includes the contribution from all matter except CDM. Hence we can define 'geometric' equations of state for CDM and other matters including massive graviton, respectively, through

$$
\begin{aligned}
H_{m}^{2} & =\frac{8 \pi G}{3 c^{2}} \rho_{m}^{0} a_{0}^{3\left(1+w_{m}^{\mathrm{geo}}\right)} a^{-3\left(1+w_{m}^{\mathrm{geo}}\right)}, \\
H_{\delta}^{2} & =\frac{8 \pi G}{3 c^{2}} \rho_{g}^{0} a_{0}^{3\left(1+w_{\delta}^{\mathrm{geo}}\right)} a^{-3\left(1+w_{\delta}^{\mathrm{geo}}\right)} .
\end{aligned}
$$

Using $x=\ln \left(a_{0} / a\right)$, we can rewrite the above two equations as

$$
\begin{aligned}
H_{m}^{2} & =\frac{8 \pi G}{3 c^{2}} \rho_{m}^{0} e^{-3 x\left(1+w_{m}^{\mathrm{geo}}\right)}, \\
H_{\delta}^{2} & =\frac{8 \pi G}{3 c^{2}} \rho_{\delta}^{0} e^{-3 x\left(1+w_{\delta}^{\mathrm{geo}}\right)}
\end{aligned}
$$

Differentiating Eq. (40) with respect to $x$, we obtain

$$
2 H_{\delta} \frac{d H_{\delta}}{d x}=-3\left(1+w_{\delta}^{\mathrm{geo}}\right) H_{\delta}^{2} .
$$

Rearranging this equation, one arrives at

$$
w_{\delta}^{\text {geo }}=-1-\frac{2}{3} \frac{1}{H_{\delta}} \frac{d H_{\delta}}{d x}=-1-\frac{1}{3} \frac{d \ln H_{\delta}^{2}}{d x} \equiv w_{\mathrm{DE}},
$$


which confirms that $w_{\mathrm{DE}}$ differs from the native $\operatorname{EoS} w_{g}^{\text {nat }}$ and equals to effective EoS $w_{g}^{\text {eff }}$ when we use Eq. (21) as Hubble parameter. Note that our $w_{\mathrm{DE}}$ is different from that (2.14) of Ref. [3] even their forms are the same because their Hubble equations are different. In addition, we can obtain effective EoS for total cosmological fluid as defined in Ref [2]:

$$
w^{\mathrm{eff}}=-1-\frac{1}{3} \frac{d \ln H^{2}}{d x}=-1+\frac{\Omega_{m}^{0}\left(\frac{a_{0}}{a}\right)^{3}+\frac{\alpha_{g}^{2}}{6} a^{2}}{\Omega_{m}^{0}\left(\frac{a_{0}}{a}\right)^{3}+\frac{\alpha_{g}^{2}}{4}\left(1-a^{2}\right)},
$$

which is depicted in Fig. 2. This EoS $w^{\text {eff }}$ is exactly the same as

$$
w_{t}=\frac{p_{t}}{\rho_{t}}
$$

which is defined in (20).

\section{INTERACTION MECHANISM}

We have revisited the Massive Graviton Dark Matter scenario (MGCDM) which was recently considered as an alternative to dark energy models. When introducing the native and effective equations of state, it was shown that there is no phantom phase in the evolution toward the far past of $a \rightarrow 0$ but the past accelerating phase appears.

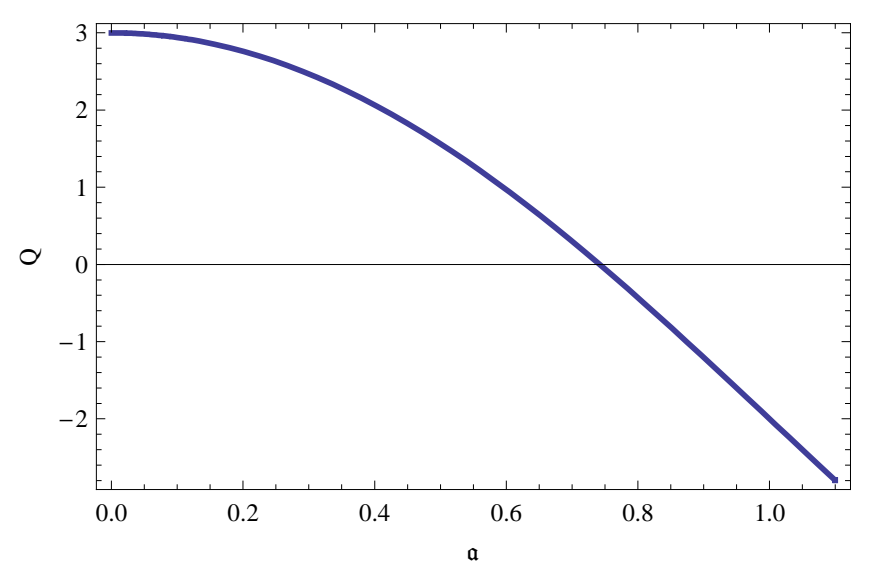

FIG. 5: Time evolution for interaction $Q$ as a function of scale factor $a$ with 1 its coefficient. We note that $Q=0$ at $a=0.74$.

How do we understand the past accelerating phase in the MGCDM? It is well known that the interaction between two different matters give rises to acceleration [10 12$]$.

In the MGCDM, the interaction between massive graviton and CDM generates acceleration in the past. Graph of Fig. 5 shows that the $\operatorname{CDM}\left(\rho_{m}, \Omega_{m}\right)$ decays to massive graviton 
$\left(\rho_{g}, \Omega_{g}\right)$ for $Q>0$ and $0 \leq a<0.74$, while massive graviton decays to CDM for $Q<0$ and $0.74<a<1$. This picture is consistent with the behavior of $\left(\Omega_{g}, \Omega_{m}\right)$ in Fig. 2. We note that the effective EoS $w_{g}^{\mathrm{eff}}$ is an decreasing function of $x$ in the backward direction as is shown in Fig. 2. On the other hand, it is noted that the native EoS $w_{g}^{\text {nat }}$ is meaningless because we are in the interacting picture. The graph of Fig. 6 shows the same graph depicted in terms of variable $x$. It shows that the CDM decays to massive graviton for $Q>0$ and $-\infty<x<0.98$, while massive graviton $\left(\rho_{g}, \Omega_{g}\right)$ decays to $\operatorname{CDM}\left(\rho_{m}, \Omega_{m}\right)$ for $Q<0$ and $0.98<x<1.16$. The cosmic anti-fraction arisen from the CDM decay process to massive graviton induces acceleration in the past universe [10 12].

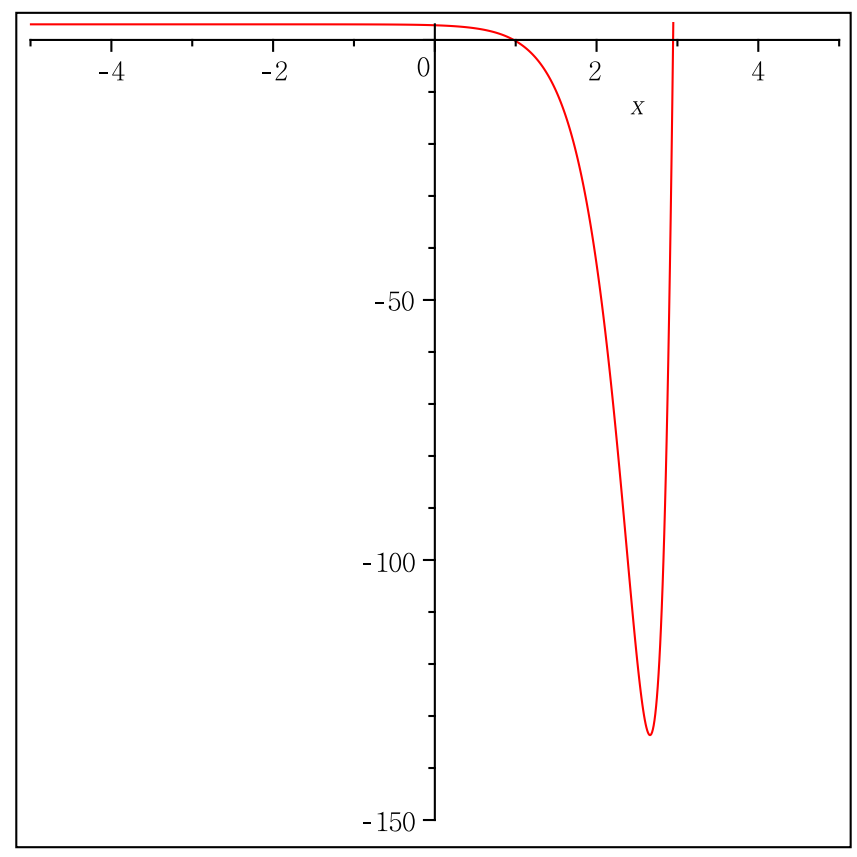

FIG. 6: Time evolution for interaction $Q$ as a function of $x$ with 1 its coefficient for $\Omega_{m}^{0}=0.27$, $\chi=3.1 \times 10^{-4}$, and $\alpha_{g}=1.8$. We note that $Q=0$ at $x=0.98$ and 2.95 , and $x=1.16(a=1)$.

Finally, we would like to mention that the condition of positive energy density and negative pressure (13) is not mandatory to be fixed. In Appendix, we can extend this condition by introducing the background static scaling factor $a_{s}$.

Consequently, any phantom phase des not appear in the evolution toward the far past. The interaction between massive graviton and CDM generates the past acceleration in the MGCDM. 


\section{Acknowledgments}

This work was supported by the National Research Foundation of Korea (NRF) grant funded by the Korea government (MEST) (No.2010-0028080).

\section{Appendix: Background static metric with scaling $a_{s}$}

If we choose the flat background metric $g_{0}$ as

$$
d s_{0}^{2}=c^{2} d t^{2}-a_{s}^{2}\left[d r^{2}+r^{2} d \Omega_{2}^{2}\right]
$$

with $a_{s}$ an arbitrary scale factor, the relevant quantities are changed as

$$
\begin{gathered}
\rho_{g}=\frac{3 m_{g}^{2} c^{6}}{32 \pi G \hbar^{2}}\left(a_{s}^{2}-a^{2}\right), \\
p_{g}=\frac{m_{g}^{2} c^{6}}{32 \pi G \hbar^{2}} a^{2}\left(a^{2}-a_{s}^{2}\right) .
\end{gathered}
$$

We note that the positive energy density and negative pressure is required to explain the accelerating universe. In this case, one has the inequality

$$
a^{2}<a_{s}^{2}
$$

Although the native EoS remains unchanged, the effective EoS $w_{g}^{\text {eff }}$ is changed as

$$
w_{g}^{\mathrm{eff}}(a)=-\frac{1-\frac{5}{3} \frac{a^{2}}{a_{s}^{2}}}{1-\frac{a^{2}}{a_{s}^{2}}}
$$

which is the same form as Eq. (19) except scaling factor $a_{s}^{2}$. Also, the current scale factor is redefined by

$$
a_{0}=\sqrt{a_{s}^{2}-\frac{4}{\alpha_{g}^{2}}\left(1-\Omega_{m}^{0}-\chi \Omega_{m}^{0}\right)} .
$$

The condition of $\alpha_{g}$ is slightly changed as

$$
\alpha_{g}>\frac{2}{a_{s}} \sqrt{1-\Omega_{m}^{0}-\chi \Omega_{m}^{0}}
$$

which corresponds to the inequality condition on the mass of massive graviton as

$$
m_{g}>\frac{2}{a_{s}} \sqrt{1-\Omega_{m}^{0}-\chi \Omega_{m}^{0}} m_{H} .
$$


That is, by introducing the scaling $a_{s}$ of the background static metric, it is possible to restrict the maximum scale factor for the universe as in (48).

[1] M. Visser, Gen. Rel. Grav. 30, 1717 (1998) arXiv:gr-qc/9705051.

[2] M. E. S. Alves, O. D. Miranda and J. C. N. de Araujo, Phys. Lett. B 700, 283 (2011) arXiv:0907.5190 [astro-ph.CO]].

[3] S. Basilakos, M. Plionis, M. E. S. Alves and J. A. S. Lima, Phys. Rev. D 83, 103506 (2011) arXiv:1103.1464 [astro-ph.CO]].

[4] W. Zimdahl and D. Pavon, Phys. Lett. B 521 (2001) 133 arXiv:astro-ph/0105479.

[5] B. Wang, Y. g. Gong and E. Abdalla, Phys. Lett. B 624 (2005) 141 arXiv:hep-th/0506069].

[6] H. Kim, H. W. Lee and Y. S. Myung, Phys. Lett. B 632 (2006) 605 arXiv:gr-qc/0509040.

[7] K. Y. Kim, H. W. Lee and Y. S. Myung, Mod. Phys. Lett. A 22 (2007) 2631 arXiv:0706.2444 $[\mathrm{gr}-\mathrm{qc}]]$.

[8] P. Wang and X. H. Meng, Class. Quant. Grav. 22, 283 (2005) arXiv:astro-ph/0408495.

[9] H. W. Lee, K. Y. Kim and Y. S. Myung, Eur. Phys. J. C 71, 1585 (2011) arXiv:1010.5556 [hep-th]].

[10] W. Zimdahl, D. J. Schwarz, A. B. Balakin and D. Pavon, Phys. Rev. D 64, 063501 (2001) arXiv:astro-ph/0009353.

[11] A. B. Balakin, D. Pavon, D. J. Schwarz and W. Zimdahl, New J. Phys. 5, 85 (2003) arXiv:astro-ph/0302150].

[12] Y. S. Myung, Phys. Lett. B 626, 1 (2005) arXiv:hep-th/0502128. 